\title{
Cellular evidence for nano-scale exosome secretion and interactions with spermatozoa in the epididymis of the Chinese soft-shelled turtle, Pelodiscus sinensis
}

\author{
Hong Chen ${ }^{1}$, Ping Yang ${ }^{1}$, Xiaoya Chu ${ }^{1}$, Yufei Huang ${ }^{1}$, Tengfei Liu ${ }^{1}$, Qian Zhang ${ }^{1}$, \\ Quanfu Li ${ }^{1}$, Lisi Hu ${ }^{1}$, Yasir Waqas ${ }^{1}$, Nisar Ahmed ${ }^{1}$ and Qiusheng Chen ${ }^{1}$ \\ ${ }^{1}$ Laboratory of Animal Cell Biology and Embryology, College of Veterinary Medicine, Nanjing Agricultural University, Nanjing, \\ Jiangsu Province, China \\ Correspondence to: Qiusheng Chen, email: chenqsh305@njau.edu.cn \\ Keywords: Chinese soft-shelled turtle; nano-scale exosomes; spermatozoa; epididymis; interaction; Pathology Section \\ Received: December 02, $2015 \quad$ Accepted: February 24, $2016 \quad$ Published: March 15, 2016
}

\section{ABSTRACT}

The epididymis is the location of sperm maturation and sperm storage. Recent studies have shown that nano-scale exosomes play a vital role during these complicated processes. Our aim was to analyze the secretory properties of epididymal exosomes and their ultrastructural interaction with maturing spermatozoa in the Chinese soft-shelled turtle. The exosome marker CD63 was primarily localized to the apices of principal cells throughout the epididymal epithelium. Identification of nano-scale exosomes and their secretory processes were further investigated via transmission electron microscopy. The epithelium secreted epididymal exosomes (50 300 $\mathrm{nm}$ in diameter) through apocrine secretion and the multivesicular body (MVB) pathway. Spermatozoa absorbed epididymal exosomes through endocytosis or membrane fusion pathways. This study shows, for the first time, that nano-scale exosomes use two secretion and two absorption pathways in the reptile, which may be contribute to long-term sperm storage.

\section{INTRODUCTION}

After spermiation in the testis, spermatozoa are functionally incompetent and are unable to fertilize an oocyte even though they are morphologically mature. Fertilization ability is acquired during spermatozoa transit through the epididymis $[1,2]$. The epididymis is a single, long, convoluted tubule that is usually divided into three main segments based on their anatomical properties: caput, corpus, and cauda [3]. Epididymal transit lasts 5-10 days in the mouse [4] and leads to the acquisition of sperm motility and the ability to recognize and fertilize oocytes [5]. Unlike mammals, most reptile species, such as snakes [6] and turtles [7], have more obvious sperm storage [8] in the epididymis. In our previous work, we found that the Chinese soft-shelled turtle Pelodiscus sinensis has typical long-term sperm storage in the epididymis $[9,10]$. Spermatogenesis ends with spermiation, when spermatozoa are released into the epididymis in a single event in late October [11]. Immature spermatozoa are transferred into the epididymis and stored until the following reproductive season (from May to October)
[12]. This reproductive strategy is necessary for P.sinensis to successfully breed.

When spermatozoa migrate to the epididymis, chromatin in the sperm head condenses and the process of transcription and translation arrests. Sperm maturation and modification require the interaction between male gamete and their microenvironment in the epididymis [13]. The epididymal fluid milieu is comprised of organic and inorganic compounds generated by epithelial secretion and reabsorption $[7,14]$. While in the epididymis, spermatozoa are exposed to different secretions that modulate their metabolism, their membrane and intracellular structures, and their biochemical composition [15]. The most important maturational changes of spermatozoa involve plasma membrane remodeling and the acquisition of forward motility [2]. These changes are primarily the result of proteins and lipids acquired by the spermatozoa plasma membrane [16, 17]. Intercellular communications are performed by membrane vesicle secretion and uptake in many biological systems. These vesicles can be classified as exosomes, ectosomes, microvesicles, apoptotic vesicles, and other extracellular vesicles 
according to their size, functions and cellular origin [18]. Exosomes are membrane-bound nano-particles secreted from cells under both physiological and pathological conditions, and they are released into the extracellular environment upon multivesicular body (MVB) fusion with the plasma membrane $[19,20]$. Exosomes have always been defined as 30-100 $\mathrm{nm}$ in diameter, but a recent study has shown that exosomes from different histological ovarian epithelial cancer cells differ in size [21]. Exosomes are conserved structures found in every cell type [22] and were identified many years ago [23]. With the exception of protein and lipid transfer, exosomes are a novel mechanism for the genetic exchange between cells by delivering mRNAs and microRNAs [24]. Extracellular vesicles and their miRNA cargo are transferred to recipient cells after endocytosis or membrane fusion [25]. Due to their ability to transfer nucleic acids, the study of extracellular vesicles in oncology is a rapidly evolving and expanding field [26-28].

Similar to other biological fluids, epididymal fluids include nano-scale exosomes that measure $50 \sim 300 \mathrm{~nm}$ in diameter. These vesicles are also called epididymosomes and are released from epididymal principal cells [29]. Epididymal exosomes play a major role in sperm maturation by transferring proteins important for acquiring fertilization ability and forward motility $[15,30]$. The presence of small membrane vesicles, or nano-scale exosomes, has been described in rats [31], mice [32], and bovine [33],but there has not yet been a report of the presence of membranous vesicles in the reptile epididymis. The majority of studies have focused on exosomes isolated from cell culture fluid and adopted in vitro models to study the mechanisms of secretion and absorption $[33,34]$.

In this study, we used the Chinese soft-shelled turtle to investigate nano-scale exosomes in sperm maturation and sperm storage. To our knowledge, this is the first in vivo experiment to substantiate the secretion pathway of epididymal exosomes in the reptile. We also examined the ultrastructural characteristics between nano-scale exosomes and maturing spermatozoa.

\section{RESULTS}

\section{CD63 expression in the epididymis}

We divided the Chinese soft-shelled turtle epididymis into three regions: caput, corpus and cauda. The epididymis was lined with pseudostratified epithelium primarily composed of principal and basal cells (Figure 1). CD63-positive cells were distributed in the caput (Figure $2 \mathrm{~A}$ ), corpus (Figure 2B) and cauda (Figure 2C). The epididymal lumen was filled with spermatozoa at different stages of sperm storage. We observed the strongest immunoreactivity at the apices of principal cells (Figure 2D). Some CD63-positive structures appeared to be shed from principal cells (Figure 2E). There were many positive particles scattered between spermatozoa (Figure 2F). CD63 expression was similar at early Nov, April and July, respectively, which corresponded to the early, intermediate and late stages of sperm storage in the epididymis.

\section{TEM detection of nano-scale epididymal exosomes}

We observed nano-scale exosomes in the lumen of all three epididymal regions (Figure 3), which ranged from 50-300 nm in diameter. We also detected them within (Figure 4A) and outside of (Figure 4C) the epithelium. Interestingly, multivesicular body (MVB) associated with nano-scale exosomes (Figure 4B) was located in the epididymal epithelial cells (Figure 4A). In addition, some apical blebs containing many nano-scale exosomes localized to the epithelial surface (Figure 5A). These blebs detached from the epithelial surface. Finally, as a consequence of fragmentation, the contents of the blebs were released into the epididymal lumen (Figure 5C).

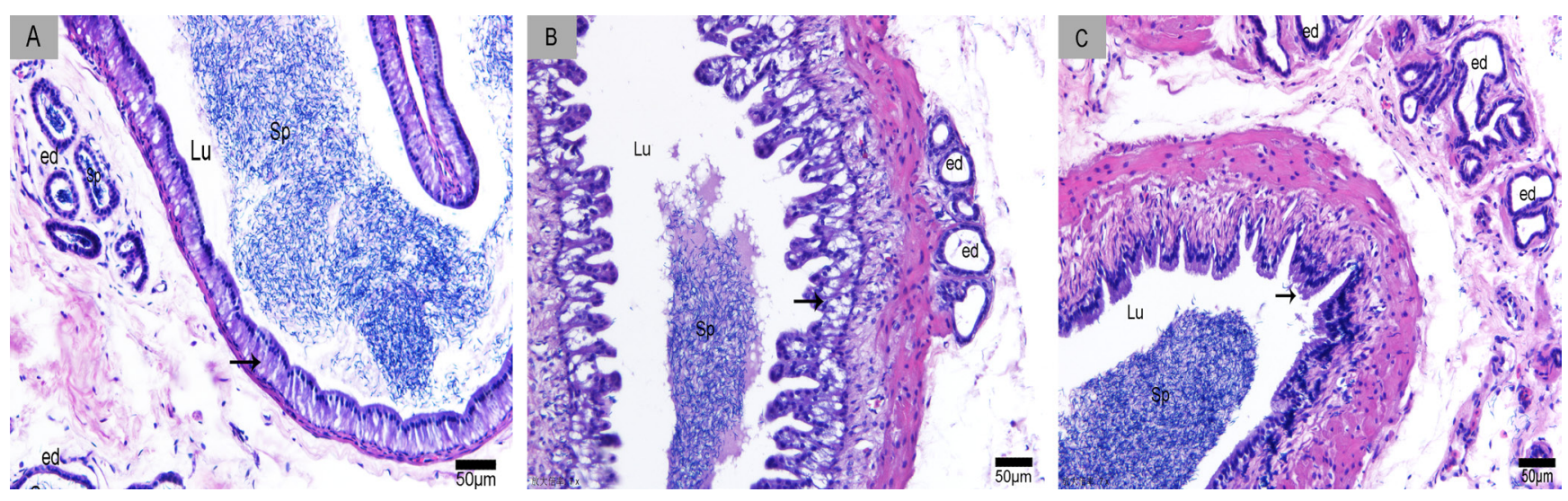

Figure 1: Sperm storage in the epididymis. A. caput, B. corpus and C. cauda. Lu: lumen; Sp: spermatozoa; arrowhead: epididymal epithelium; ed: efferent duct; H \& E stain; Bars: A, B, C = 50 $\mu \mathrm{m}$. 

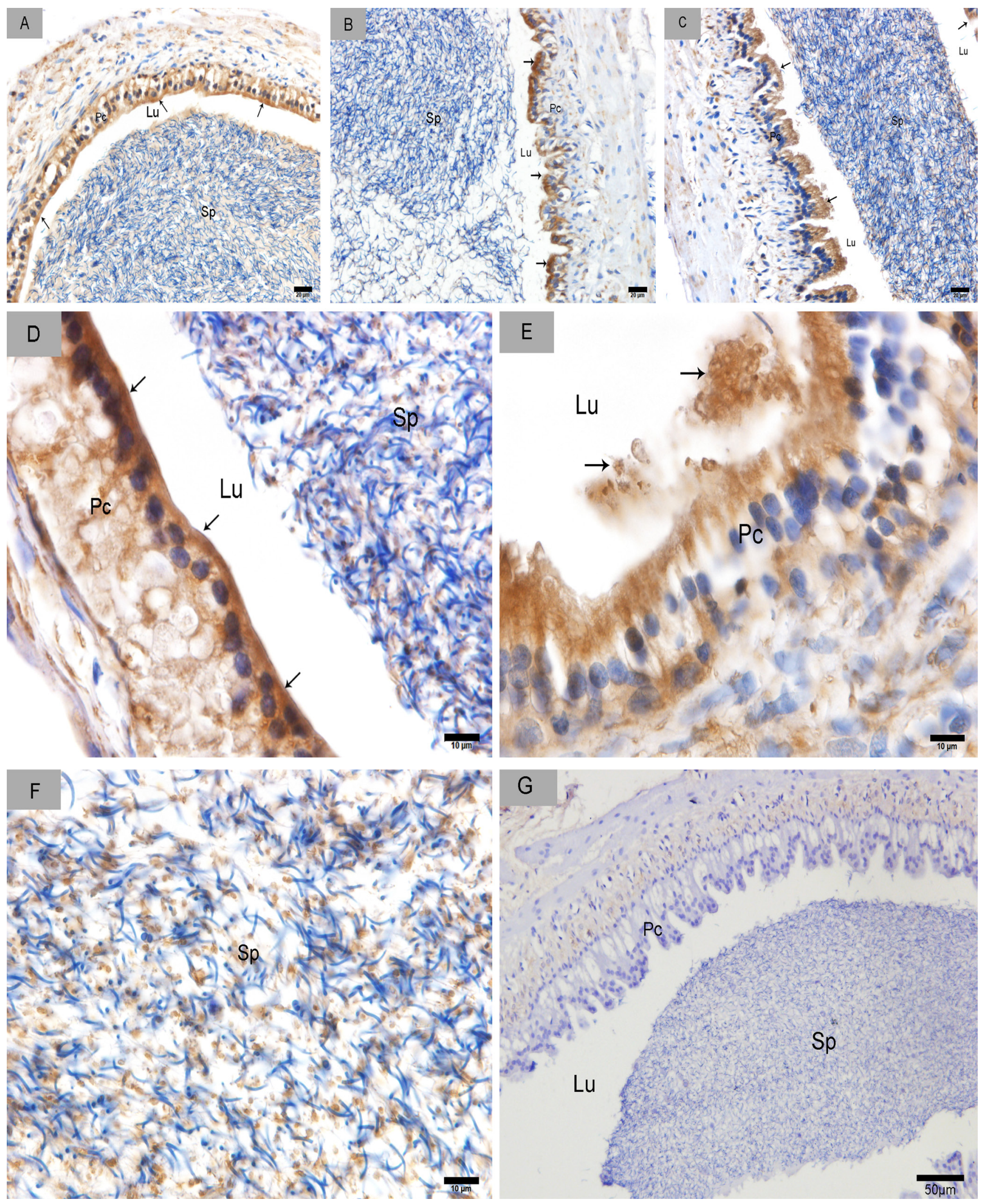

Figure 2: CD63 immunohistochemistry in the epididymis. Arrowhead shows positive CD63 expression. A. caput (Nov), B. corpus (Apr), C. cauda (July), D. caput (Nov), E. corpus (Apr), F. cauda (July) and G. Negative control. Sp: spermatozoa; Lu: lumen; Pc: Principal cell; Bars: A, B, C $=20 \mu \mathrm{m} ; \mathrm{D}, \mathrm{E}, \mathrm{F}=10 \mu \mathrm{m} ; \mathrm{G}=50 \mu \mathrm{m}$. 

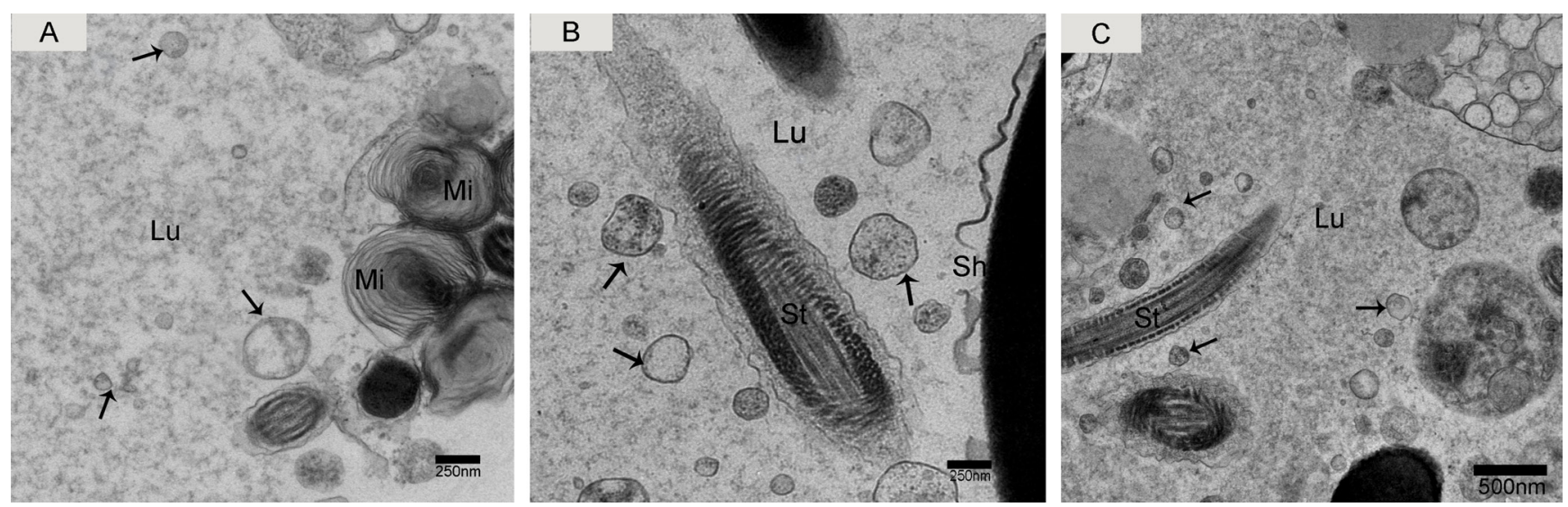

Figure 3: Electron micrograph of nano-scale exosomes (arrowhead) around spermatozoa in the epididymal lumen. A. caput, B. corpus, C. cauda. Lu: lumen; Mi: mitochondria; St: sperm tail; Sh: sperm head; Bars: A, B $=250 \mathrm{~nm}$; C = $500 \mathrm{~nm}$.
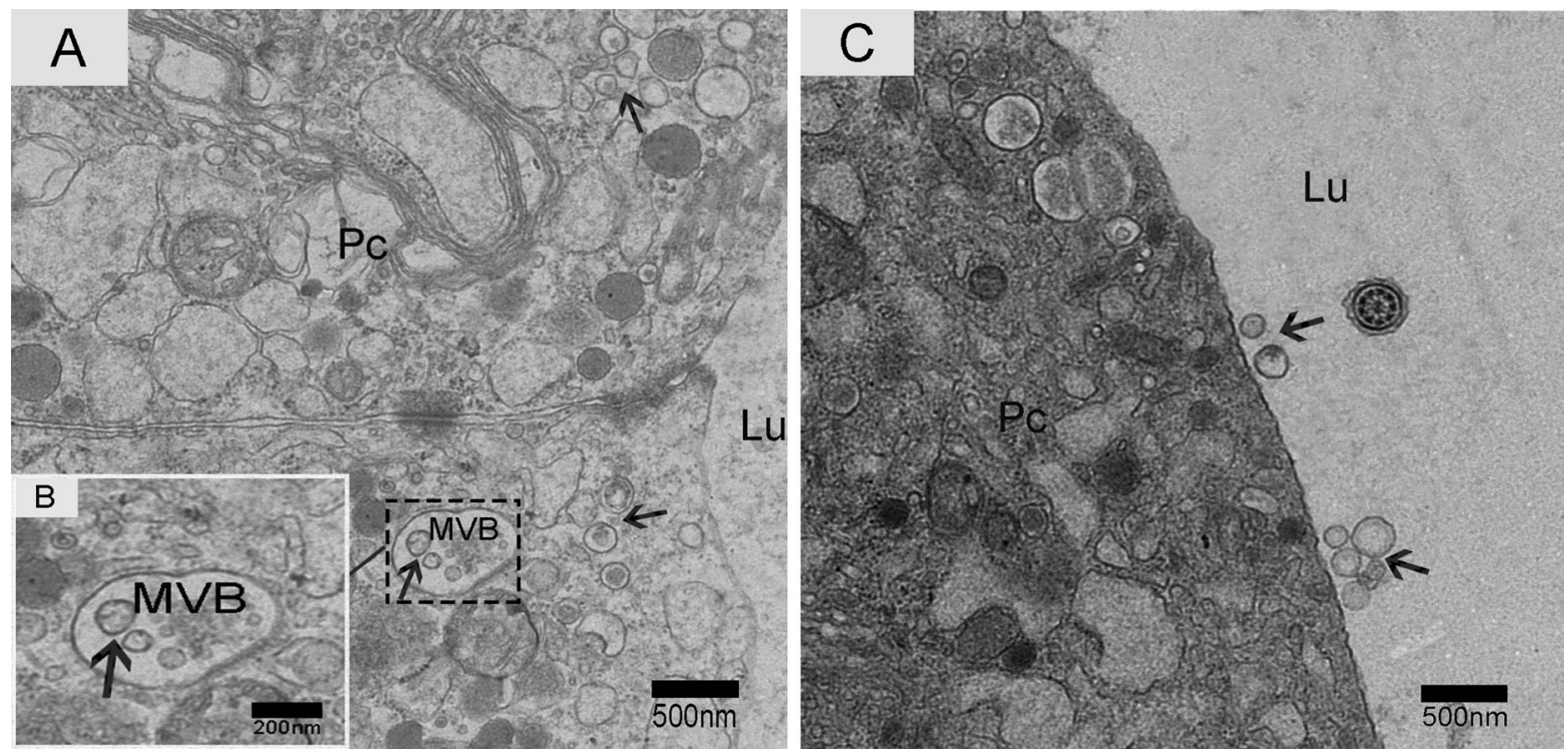

Figure 4: Electron micrograph of nano-scale exosomes. Nano-scale exosomes (arrowhead) are scattered in the principal cells or enclosed in MVB. A. Zoomed-in image of MVB containing nano-scale exosomes. B. Nano-scale exosomes (arrowhead) are close to the principal cell surface. C. Pc: principal cell; MVB: multivesicular body; Lu: lumen; Bars: A, C=500 nm; B = $200 \mathrm{~nm}$.

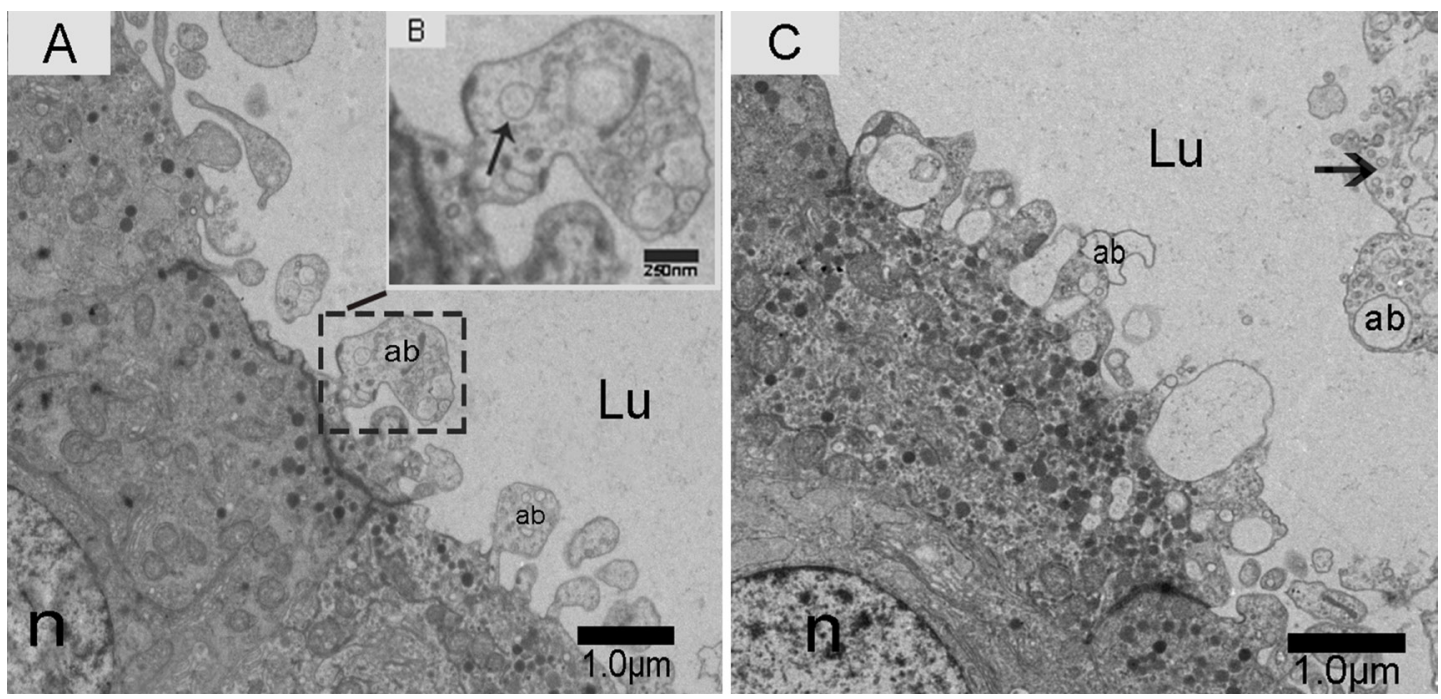

Figure 5: Electron micrograph of nano-scale exosomes (arrowhead) released by the epithelium. Apical blebs formed on the principal cell surface. A. Zoomed-in image of apical blebs containing nano-scale exosomes (arrowhead). B. Nano-scale exosomes (arrowhead) are released into the lumen. C. Lu: lumen; n: nucleus; ab: apical blebs; Bars: A, C =1.0 $\mu \mathrm{m}$; B $=250 \mathrm{~nm}$. 


\section{Interactions between nano-scale exosomes and spermatozoa in the epididymis}

Nano-scale exosomes were distributed among spermatozoa throughout the lumen of the epididymis, and some made contact with the sperm head (Figure $6 \mathrm{~A})$. When the nano-scale exosomes were next to the spermatozoa midpiece, the corresponding cytoplasm membrane was depressed (Figure 6B). We also observed that the principal piece membrane in maturing spermatozoa morphologically contacted with the nanoscale exosomes within the lumen (Figure 6C). Some nanoscale exosomes localized to spermatozoa cytoplasm and the lumen, and these nano-scale exosomes showed a clear double-layer membrane structure (Figure 7A). The nanoscale exosome membrane fused with the spermatozoa membrane, and sometimes the entire nano-scale exosome was internalized by the spermatozoa via endocytosis (Figure 7B).

\section{DISCUSSION}

Cell-cell communication is a very complicated mechanism for information exchange. Direct membrane contact is the most evident way for two cells to communicate [35]. However, some soluble secreted molecules have also been shown to be important
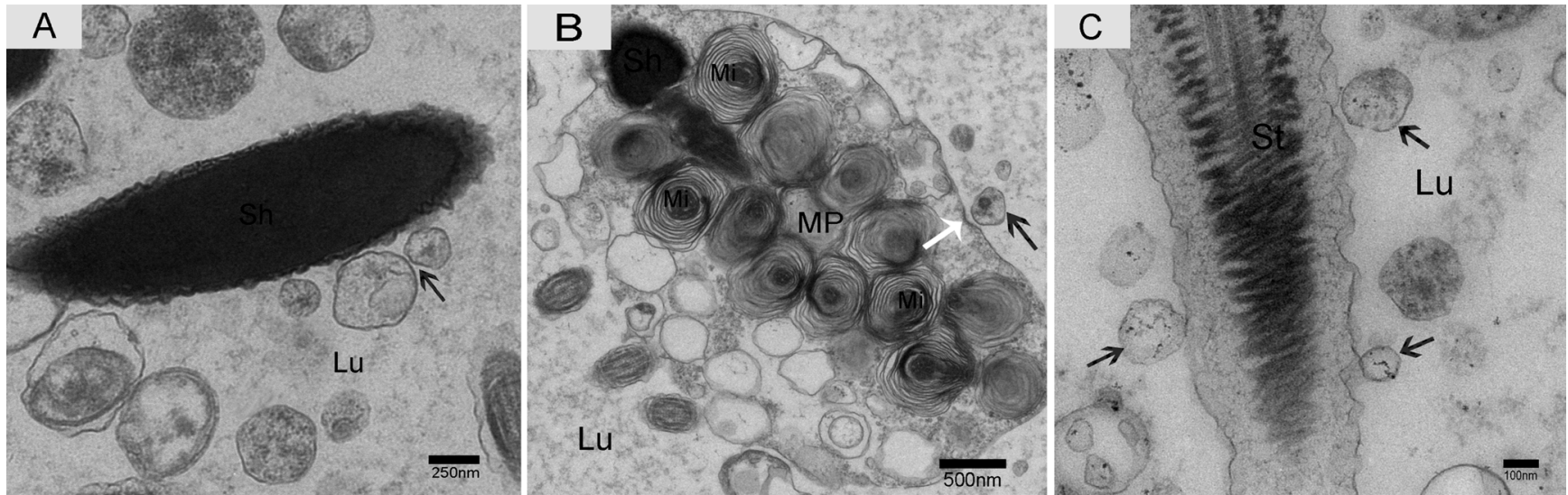

Figure 6: Electron micrograph of nano-scale exosomes and spermatozoa in the epididymis. Nano-scale exosomes (arrowhead) contacted the spermatozoa head A., midpiece B. and principal piece $\mathbf{C}$. The corresponding cytoplasmic membrane (white arrow) is depressed (B). Lu: lumen; Sh: sperm head; MP: midpiece; St: sperm tail; Mi: mitochondria; Bars: A= $250 \mathrm{~nm}, \mathrm{~B}=500 \mathrm{~nm}, \mathrm{C}=$ $100 \mathrm{~nm}$.
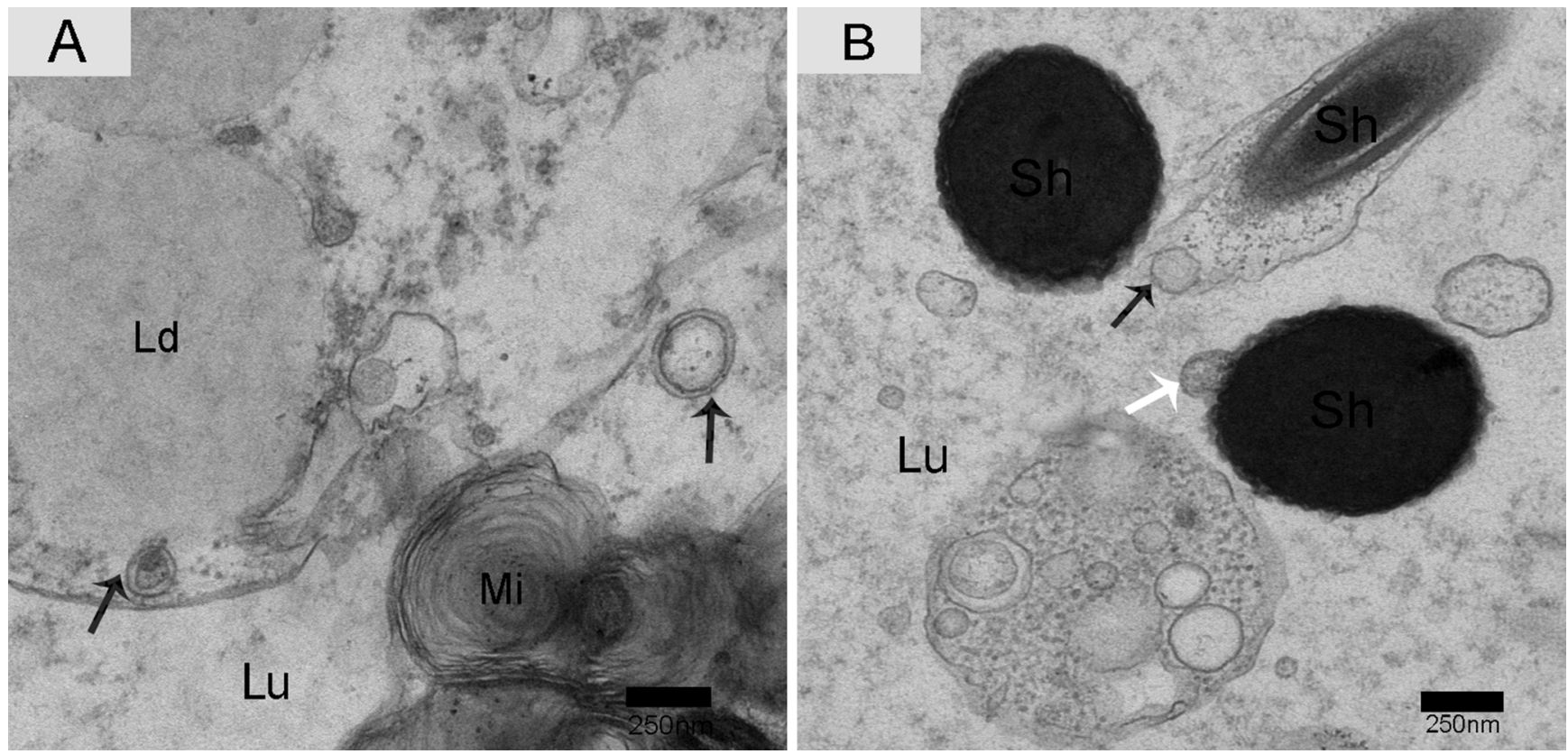

Figure 7: Electron micrograph of nano-scale exosomes with double-layer membranes and absorption in the epididymis. Nano-scale exosomes (arrowhead) have a double-layered membrane. A. Nano-scale exosomes were taken up by the endocytic pathway (arrowhead) or through membrane fusion (white arrow). B. Lu: lumen; Sh: sperm head; Mi: mitochondria; Ld: lipid droplets; Bars: A, B $=250 \mathrm{~nm}$. 
mediators of information transmission, including cytokines, hormones, and bioactive lipids. An increasing number of studies have shown a third mechanism for cell-cell communication where exosomes (30 100 nm in diameter) released by one cell can transmit essential information to its target cells [36-38]. Thus, all nano-scale exosomes secreted from cells under both physiological and pathological conditions should be recognized as a new modulatory system, just like the neural and humoral regulation systems. Here, we used the Chinese soft-shelled turtle epididymis as a model to explore the roles of nanoscale exosomes during sperm storage and maturation. Our results revealed that nano-scale exosomes can constitute the microenvironment of the epididymis to allow for longterm sperm storage, which is important for each step of sperm maturation in the epididymis.

The epididymis has an extraordinary complexity at both the structural and functional levels [39]. The segmented gene expression patterns throughout the epididymal duct of mammals results in distinct secretions in the intraluminal milieu and the formation of microenvironments optimal for each step of sperm maturation [40]. Thus, the three epididymal segments can secrete different exosomes into the lumen of mammals. Our previous study showed that the efferent ducts are present throughout all epididymal regions, which is a typical feature of the Chinese soft-shelled turtle [9]. If the epididymis is important in sperm storage and maturation, then the spermatozoa would be in the efferent ducts down the entire length of the epididymis. The three epididymal segments have similar abilities in sperm storage, which is distinct from mammals. In this study, we found that CD63 expression was similar in each of the three epididymal regions. This is consistent with the ability of each epididymal segment to store sperm. The epithelium releases different size nano-scale exosomes to facilitate spermatozoa maturation at different stages of sperm storage. This is likely why we saw similar CD63 expression at the three time points.

Only one secretion pathway involved in nanoscale exosomes has been reported in the mammalian epididymis. Previous analysis revealed that the epididymal epithelium was capable of apocrine secretion [41]. Apocrine secretion is characterized by the formation of apical blebs by the epithelium, where the apical blebs detach from the apical membrane and release their contents into the lumen through fragmentation [42-44]. We performed TEM analysis, which is considered the gold standard for exosome identification $[45,46]$. We found that the epididymal exosomes in the Chinese soft-shelled turtle were round in shape and approximately 50-300 nm in size, which was similar to those previously described in mammals [33, 42, 47]. Importantly, TEM results provided significant evidence for apocrine secretion of nano-scale exosomes. We also detected multivesicular body (MVB) in the principal cells of Chinese soft-shelled turtles. MVB fusion with the plasma membrane led to the release of the internal vesicles into the extracellular space. There was a clear significant difference between the above two nano-scale exosome secretion pathways. Based on these observations, we hypothesized that the principal cells secrete nano-scale exosomes into the epididymal lumen by apocrine secretion and MVB pathways.

There are currently two known mechanisms of exosome generation in tumour cells. The first model is that exosomes formed via the endocytic pathway in a two-step process and are released into the extracellular environment from the plasma membrane via multivesicular body (MVB) [48, 49]. The second means of secretion is the ESCRT-independent mechanism [50], and this method was shown to require the sphingolipid ceramide (Cer) [51]. Therefore, our results provide a new research direction for tumour-derived exosomes.

Information transfer can occur through several mechanisms, while direct contact is a very important basic method [24]. The ultrastructure in the Chinese soft-shelled turtle indicates that nano-scale exosomes contact the head, midpiece and principal piece of spermatozoa within the epididymal lumen. TEM results gave clear evidence for two different absorption pathways of nano-scale exosomes. One pathway involves nano-scale exosome membrane fusion within the spermatozoa membrane, and the other pathway is through the endocytic pathway (as shown in Figure 7B). Analysis of their interaction and absorption could provide direct evidence for the roles of epididymal exosomes, which may act as messengers for epithelium-spermatozoa communication and signal transduction. Meanwhile, nano-scale exosome absorption and contact with different regions of spermatozoa are very important factors for long-term sperm storage in the epididymis of Chinese soft-shelled turtles.

In summary, the apical surface of epididymal epithelial cells secreted nano-scale exosomes into the luminal fluids. Furthermore, principal cells released epididymal exosomes through apocrine secretion and MVB pathways. We provided significant evidence for the contact and absorption of nano-scale exosomes by spermatozoa. Chinese soft-shelled turtle may be an in vivo model for investigating and obtaining nano-scale exosomes.

\section{MATERIALS AND METHODS}

\section{Animals}

The Chinese soft-shelled turtle Pelodiscus sinensis is one of the most representative classes of reptiles that hibernate (from Dec to Apr). Sexually mature male Chinese soft-shelled turtles were purchased in early November, April and July from a pond in Nanjing, south 
China. Five turtles were taken at random from the pond each time, anaesthetized by intraperitoneal administration of sodium pentobarbital $(20 \mathrm{mg} / \mathrm{kg}$ ) and sacrificed by cervical dislocation. Five epididymis pairs were used each time, and all efforts were made to minimize animal suffering. The protocol was approved by the Science and Technology Agency of Jiangsu Province (SYXK (SU) 2010-0005).

\section{Light microscopy}

Samples were fixed in $10 \%$ neutral buffered formalin (v/v) overnight, embedded in paraffin wax, and serial sectioned $(5 \mu \mathrm{m})$. A section of each region of the epididymis (caput, corpus and cauda) was stained with haematoxylin and eosin (H \& E) for light microscopic observation.

\section{Immunohistochemistry}

Three different month epididymis of the Chinese soft-shelled turtle were examined by immunohistochemistry, which showed surface protein CD63 expression on exosomes, as it is a commonly used exosome marker [52]. Three tissue slides containing 5 $\mu \mathrm{m}$ sections of the epididymis (caput, corpus and cauda) were processed through a standard immunohistochemistry protocol as described previously [53]. The rabbit antiCD63 antibody (ab134045, Abcam, Cambridge, UK) was used at a dilution of 1:75. Sections incubated in PBS alone served as negative controls.

\section{Transmission electron microscopy (TEM)}

Three epididymal sample regions were cut into small blocks and fixed in $2.5 \%(\mathrm{v} / \mathrm{v})$ glutaraldehyde in phosphate-buffered saline (PBS; $4{ }^{\circ} \mathrm{C}, \mathrm{pH} 7.4,0.1 \mathrm{M}$ ) for $24 \mathrm{~h}$. The samples were rinsed in PBS, post-fixed in $1 \%(\mathrm{w} / \mathrm{v})$ osmium tetroxide in the same buffer for 1 $\mathrm{h}$ and washed in buffer. The samples were dehydrated in increasing concentrations of ethyl alcohol and infiltrated with a propylene oxide-Araldite mixture for embedding in Araldite. The blocks were sectioned, and ultrathin sections $(50 \mathrm{~nm})$ were mounted on Formvar-coated grids, and stained with uranyl acetate and lead citrate for $20 \mathrm{~min}$ per step. The sections were examined and photographed using a transmission electron microscope (TEM; Hitachi H-7650, Japan).

\section{ACKNOWLEDGMENTS}

This work was supported by the National Natural Science Foundation of China (grant number 31272521) and Priority Academic Program Development of Jiangsu
Higher Education Institutions, PAPD.

\section{CONFLICTS OF INTERESTS}

The authors declare no competing financial interests.

\section{Authors' contributions}

Hong Chen designed the experiments and drafted the manuscript. Ping Yang, Xiaoya Chu, Yufei Huang, Tengfei Liu, Qian Zhang, Quanfu Li, Lisi Hu, Yasir Waqas and Nisar Ahmed participated in the study design and performed data analysis. Prof.Q. Chen conceived the study and participated in its design and coordination and helped draft the manuscript. All authors read and approved the final manuscript.

\section{REFERENCES}

1. Cooper T. Role of the epididymis in mediating changes in the male gamete during maturation. Tissue ReninAngiotensin Systems (Springer). 1995; pp. 87-101.

2. Jones R. Plasma membrane structure and remodelling during sperm maturation in the epididymis. Journal of reproduction and fertility Supplement. 1997; 53:73-84.

3. Thimon Vr, Koukoui Od, Calvo Eq and Sullivan R. Region-specific gene expression profiling along the human epididymis. Molecular human reproduction. 2007; 13:691704.

4. Robaire B, Hinton BT and Orgebin-Crist M-C. The epididymis. Knobil and Neill's physiology of reproduction. 2006; 1:1071-1148.

5. Belleannée C, Calvo É, Caballero J and Sullivan R. Epididymosomes convey different repertoires of microRNAs throughout the bovine epididymis. Biology of reproduction. 2013; 89:30.

6. Almeida-Santos SM, Laporta-Ferreira IL, Antoniazzi MM and Jared C. Sperm storage in males of the snake Crotalus durissus terrificus (Crotalinae: Viperidae) in southeastern Brazil. Comparative Biochemistry and Physiology Part A: Molecular \& Integrative Physiology. 2004; 139:169-174.

7. Gist DH, Dawes SM, Turner TW, Sheldon S and Congdon J. Sperm storage in turtles: a male perspective. Journal of experimental zoology. 2002; 292:180-186.

8. Sharifi M, Ghorbani R, Fazeli A and Holt W. Evidence of sperm storage in Pipistrellus kuhlii (Chiroptera: Vespertilionidae) in western Iran. Folia zoologica. 2004; 53:1-6.

9. Bian X, Zhang L, Yang L, Yang P, Ullah S, Zhang Q and Chen Q. Ultrastructure of epididymal epithelium and its interaction with the sperm in the soft-shelled turtle Pelodiscus sinensis. Micron. 2013; 54:65-74.

10. Zhang L, Yang P, Bian X, Zhang Q, Ullah S, Waqas Y, Chen X, Liu Y, Chen W and Le Y. Modification of 
sperm morphology during long-term sperm storage in the reproductive tract of the Chinese soft-shelled turtle, Pelodiscus sinensis. Scientific reports. 2015; 5:16096.

11. Zhang L, Han XK, Li MY, Bao HJ and Chen QS. Spermiogenesis in Soft-Shelled Turtle, Pelodiscus sinensis. The Anatomical Record. 2007; 290:1213-1222.

12. Bian X, Gandahi JA, Liu Y, Yang P, Liu Y, Zhang L, Zhang $\mathrm{Q}$ and Chen $\mathrm{Q}$. The ultrastructural characteristics of the spermatozoa stored in the cauda epididymidis in Chinese soft-shelled turtle Pelodiscus sinensis during the breeding season. Micron. 2013; 44:202-209.

13. Cooper $\mathrm{T}$. Interactions between epididymal secretions and spermatozoa. Journal of reproduction and fertility Supplement. 1997; 53:119-136.

14. Dacheux J-L and Dacheux F. Protein secretion in the epididymis. The Epididymis: From Molecules to Clinical Practice (Springer). 2002; pp. 151-168.

15. Sullivan R and Saez F. Epididymosomes, prostasomes, and liposomes: their roles in mammalian male reproductive physiology. Reproduction. 2013; 146:R21-R35.

16. Olson GE, NagDas SK and Winfrey VP. Structural differentiation of spermatozoa during post-testicular maturation. The epididymis: from molecules to clinical practice (Springer). 2002; pp. 371-387.

17. Jones R. Plasma membrane composition and organisation during maturation of spermatozoa in the epididymis. The Epididymis: From Molecules to Clinical Practice (Springer). 2002; pp. 405-416.

18. Tannetta D, Dragovic R, Alyahyaei Z and Southcombe J. Extracellular vesicles and reproduction-promotion of successful pregnancy. Cellular \& molecular immunology. 2014; 11:548-63.

19. van Niel G, Porto-Carreiro I, Simoes S and Raposo G. Exosomes: a common pathway for a specialized function. Journal of biochemistry. 2006; 140:13-21.

20. Saleem SN and Abdel-Mageed AB. Tumor-derived exosomes in oncogenic reprogramming and cancer progression. Cellular and Molecular Life Sciences. 2015; 72:1-10.

21. Zhang W, Peng P, Kuang Y, Yang J, Cao D, You Y and Shen K. Characterization of exosomes derived from ovarian cancer cells and normal ovarian epithelial cells by nanoparticle tracking analysis. Tumor Biology. 2015:1-9.

22. Braicu C, Tomuleasa C, Monroig P, Cucuianu A, BerindanNeagoe I and Calin G. Exosomes as divine messengers: are they the Hermes of modern molecular oncology. Cell Death Differ. 2015; 22:34-45.

23. Piper RC and Katzmann DJ. Biogenesis and function of multivesicular bodies. Annual review of cell and developmental biology. 2007; 23:519.

24. Valadi H, Ekström K, Bossios A, Sjöstrand M, Lee JJ and Lötvall JO. Exosome-mediated transfer of mRNAs and microRNAs is a novel mechanism of genetic exchange between cells. Nature cell biology. 2007; 9:654-659.
25. Belleannée C. Extracellular microRNAs from the epididymis as potential mediators of cell-to-cell communication. Asian journal of andrology. 2015; 17:730.

26. Sousa D, Lima RT and Vasconcelos MH. Intercellular Transfer of Cancer Drug Resistance Traits by Extracellular Vesicles. Trends in molecular medicine. 2015; 21:595-608.

27. Zhang L, Zhang S, Yao J, Lowery FJ, Zhang Q, Huang W-C, Li P, Li M, Wang X and Zhang C. Microenvironmentinduced PTEN loss by exosomal microRNA primes brain metastasis outgrowth. Nature. 2015; 527:100-4.

28. Schorey JS and Bhatnagar S. Exosome function: from tumor immunology to pathogen biology. Traffic. 2008; 9:871-881.

29. Hermo L and Jacks D. Nature's ingenuity: bypassing the classical secretory route via apocrine secretion. Molecular reproduction and development. 2002; 63:394-410.

30. Sullivan R, Frenette G and Girouard J. Epididymosomes are involved in the acquisition of new sperm proteins during epididymal transit. Asian journal of andrology. 2007; 9:483-491.

31. Grimalt P, Bertini F and Fornes M. High-affinity sites for beta-D-galactosidase on membrane-bound vesicles isolated from rat epididymal fluid. Systems Biology in Reproductive Medicine. 2000; 44:85-91.

32. Johnston DS, Jelinsky SA, Bang HJ, DiCandeloro P, Wilson E, Kopf GS and Turner TT. The mouse epididymal transcriptome: transcriptional profiling of segmental gene expression in the epididymis. Biology of reproduction. 2005; 73:404-413.

33. Schwarz A, Wennemuth G, Post H, Brandenburger $\mathrm{T}$, Aumüller $\mathrm{G}$ and Wilhelm B. Vesicular transfer of membrane components to bovine epididymal spermatozoa. Cell and tissue research. 2013; 353:549-561.

34. Lai RC, Arslan F, Lee MM, Sze NSK, Choo A, Chen TS, Salto-Tellez M, Timmers L, Lee CN and El Oakley RM. Exosome secreted by MSC reduces myocardial ischemia/ reperfusion injury. Stem cell research. 2010; 4:214-222.

35. Klöhn P-C, Castro-Seoane R and Collinge J. Exosome release from infected dendritic cells: A clue for a fast spread of prions in the periphery? Journal of Infection. 2013; 67:359-368.

36. Johnstone RM. Exosomes biological significance: a concise review. Blood Cells, Molecules, and Diseases. 2006; $36: 315-321$

37. Théry C, Ostrowski M and Segura E. Membrane vesicles as conveyors of immune responses. Nature Reviews Immunology. 2009; 9:581-593.

38. Cocucci E, Racchetti G and Meldolesi J. Shedding microvesicles: artefacts no more. Trends in cell biology. 2009; 19:43-51.

39. Belleann"|e Cm, Thimon Vr and Sullivan R. Region-specific gene expression in the epididymis. Cell and tissue research. 2012; 349:717-731.

40. Dacheux J-L, Belleannée C, Guyonnet B, Labas V, TeixeiraGomes A-P, Ecroyd H, Druart X, Gatti J-L and Dacheux F. 
The contribution of proteomics to understanding epididymal maturation of mammalian spermatozoa. Systems biology in reproductive medicine. 2012; 58:197-210.

41. Sullivan R, Saez F, Girouard J and Frenette G. Role of exosomes in sperm maturation during the transit along the male reproductive tract. Blood Cells, Molecules, and Diseases. 2005; 35:1-10.

42. Sullivan R, D'Amours O, Caballero J and Belleannée C. The sperm journey in the excurrent duct: functions of microvesicles on sperm maturation and gene expression along the epididymis. Official journal of the Brazilian College of Animal Reproduction. 2015; 12:88-92.

43. Aumüller G, Renneberg H, Schiemann P-J, Wilhelm B, Seitz J, Konrad L and Wennemuth G. . The role of apocrine released proteins in the post-testicular regulation of human sperm function. The Fate of the Male Germ Cell (Springer). 1997; pp. 193-219.

44. Aumüller G, Wilhelm B and Seitz J. Apocrine secretionfact or artifact? Annals of Anatomy-Anatomischer Anzeiger. 1999; 181:437-446.

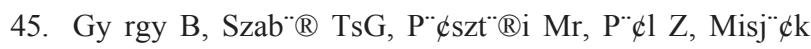
P, Aradi B1, L" $\phi s z l{ }^{\circledR}$ Vr, P" ${ }^{\circ}$ llinger E, Pap E and Kittel A. Membrane vesicles, current state-of-the-art: emerging role of extracellular vesicles. Cellular and molecular life sciences. 2011; 68:2667-2688.

46. da Silveira JC, Veeramachaneni DR, Winger QA, Carnevale EM and Bouma GJ. Cell-secreted vesicles in equine ovarian follicular fluid contain miRNAs and proteins: a possible new form of cell communication within the ovarian follicle. Biology of reproduction. 2012; 86:71.
47. Caballero JN, Frenette G, Belleannée C and Sullivan R. CD9-positive microvesicles mediate the transfer of molecules to bovine spermatozoa during epididymal maturation. 2013; 8:e65364.

48. Robbins PD and Morelli AE. Regulation of immune responses by extracellular vesicles. Nature Reviews Immunology. 2014; 14:195-208.

49. Brinton LT, Sloane HS, Kester M and Kelly KA. Formation and role of exosomes in cancer. Cellular and Molecular Life Sciences. 2015; 72:659-671.

50. Frydrychowicz M, Kolecka-Bednarczyk A, Madejczyk M, Yasar S and Dworacki G. Exosomes-Structure, Biogenesis and Biological Role in Non-Small-Cell Lung Cancer. Scandinavian journal of immunology. 2015; 81:2-10.

51. Kosaka N, Iguchi H, Yoshioka Y, Takeshita F, Matsuki $\mathrm{Y}$ and Ochiya T. Secretory mechanisms and intercellular transfer of microRNAs in living cells. Journal of Biological Chemistry. 2010; 285:17442-17452.

52. Kulshreshtha A, Ahmad T, Agrawal A and Ghosh B. Proinflammatory role of epithelial cell-derived exosomes in allergic airway inflammation. Journal of Allergy and Clinical Immunology. 2013; 131:1194-1203. e1114.

53. Waqas MY, Lisi H, Yang P, Ullah S, Zhang L, Zhang Q, Li Q, Ahmad N, Chen W and Zeshan B. Novel cellular evidence of oviduct secretions in the Chinese soft-shelled turtle Pelodiscus sinensis. Journal of Experimental Zoology Part A: Ecological Genetics and Physiology. 2015; 323:65565. 